\title{
CRH, a placental clock and preterm labour
}

\author{
The existence of a placental clock controlling the term of pregnancy has long been hypothesized. \\ Now scientists may have uncovered what makes it tick (pages 460-463).
}

Preterm labour is the major problem encountered in obstetrical practice, occurring in 7 to 10 per cent of all pregnancies and accounting for approximately 70 per cent of early neonatal mortality and morbidity. In the United States, the financial cost of caring for preterm babies has been estimated at five to six billion US\$ annually. And the emotional cost to families affected by this condition can be tremendous.

Many hypotheses have been forwarded to explain the control of term and preterm labour ${ }^{1,2}$. The current view is that term labour is effected by paracrine mechanisms within intrauterine tissues. Preterm labour does not necessarily involve mechanisms similar to those operative at term, and a consensus is growing that this condition may result from interaction of one or more factors. Among these it has been suggested that elevations in maternal plasma concentrations of corticotrophinreleasing hormone $(\mathrm{CRH})$ occur in patients with preterm labour ${ }^{3}$. Others have reported that a fall in maternal plasma $\mathrm{CRH}$ binding protein (CRH-BP) concentrations occurs at term and preterm ${ }^{4}$.

The study by McLean and colleagues in this issue of Nature Medicine ${ }^{5}$ is the first to report both measurements in the same series of patients. The particularly novel aspect of their study is the demonstration that maternal plasma CRH concentrations are elevated early in pregnancy in those patients destined to deliver preterm, and that are lower in patients destined to deliver post dates. Because the placenta is a major source of circulating $\mathrm{CRH}$ in pregnancy, the authors suggest that these results can be interpreted as reflecting the activity of a 'placental clock'.

The concept of a placental clock controlling gestation length is, in itself, not new. In rhesus monkeys, after removal of the fetus, the placenta can be delivered after a period of time similar to normal gestation length. Recently, Thorburn and colleagues ${ }^{6}$ provided strong evidence that, in pregnant sheep, prostaglandin E2 (PGE2) is secreted by the placenta into the fetal compartment to influence a wide range of fetal activities. Some fetal effects, of PGE2 may be inhibitory, preventing expression of characteristics that would be undesirable in utero but necessary after birth. PGE2 influ-

\section{John R. G. Challis}

ence declines with delivery of the placenta. Thus, placental PGE2 may inhibit fetal breathing movements during pregnancy, conserving energy while the fetus receives oxygen across the placenta. This ends at birth, with the onset of extra-uterine breathing. During late pregnancy, an increase in placental PGE2 synthesis - and secretion into the fetus - contributes to the drive to the fetal adrenal gland and, in turn, increased fetal adrenal cortisol output, which in sheep is the trigger to birth.

Placental CRH may play a similar role in human pregnancy. In addition to its paracrine actions on decidual and myometrial tissue (resulting in increased prostaglandin production and uterine contractility), $\mathrm{CRH}$ is secreted into the fetus as well as to the mother (as shown by McLean and colleagues). Placental CRH expression is higher in conditions of utero-placental compromise, such as in preeclampsia ${ }^{7}$. The possibility has been raised that its output increases in response to stressors such as hypoxemia. Because CRH is a powerful vasodilator in the placenta, its production may reflect an autoregulatory attempt to increase blood flow and to maintain fetal oxygenation. If the attempt at correction fails, placental CRH is thought to promote activity of the fetal pituitary-adrenal axis, and increased fetal cortisol secretion. However, glucocorticoids upregulate CRH gene expression in the human placenta. This activates a 'feed-forward' cascade of events that results in the delivery of the fetus from an unfavourable environment ${ }^{8}$.

The study of Mclean et al..$^{5}$ shows an inverse relationship between plasma $\mathrm{CRH}$ and CRH-BP concentrations in late pregnancy. This should lead to an increase in levels of biologically active CRH not only in the maternal circulation, but also in the placenta and maternal decidua, since these are sites of CRH-BP production. CRH-BP levels fall in response to elevations in CRH. At present it is unclear whether the primary determinant of activity is a change in $\mathrm{CRH}$ or in its binding protein, paralleling the IGF-IGFBP system. Further studies of this relationship are clearly warranted.

The clinical management of preterm labour is often empirical and of limited efficacy, because the underlying causes of the condition are generally unknown. Furthermore, the causes are likely to vary from patient to patient, making early diagnosis difficult. Raised maternal CRH values have been reported in preterm labour patients, but interpatient variation argued against clinical usefulness. Previous studies have suggested that preterm labour in the setting of infection is not associated with elevations of maternal plasma $\mathrm{CRH}^{9}$, or of placental CRH mRNA levels, emphasizing the differences in aetiology. In contrast, the findings of the present study are remarkably consistent. The incidence of preterm labour is lower than in many centres, perhaps reflecting homogeneity of the local study population. However, the early and sustained rise in plasma CRH of patients destined to deliver preterm is striking. The provocative nature of these results and their potential application to a major clinical problem certainly justifies re-evaluating previous studies, and, perhaps, extending the current observations to a prospective clinical trial.

1. Challis, J.R.G. \& Mitchell, M.D. Basic mechanisms of pre-term labour. Res, clin. Forums 16,39-58 (1994).

2. Casey, M.L. \& MacDonald, P.C. The role of prostaglandins in labour. in NIH Workshop on Initiation of Labour (eds MacDonald, P.C., Challis, J.R.G., Roberts, J.M. \& Nathanielsz, P.W.) 141-156 (Perinatology Press, Ithaca, New York, 1988).

3. Campbell, E.A. et al. Plasma corticotropin-releasing hormone concentrations during pregnancy and parturition. J. clin. Endocrin. Metabol. 64, 1054-1059 (1987).

4. Perkins, A.V., Eben, F., Wolfe, C.D.A., Schulte, H.M. \& Linton, E.A. Plasma measurements of corticotrophin-releasing hormone-binding protein in normal and abnormal human pregnancy. $J$. Endocrin. 138, 149-157 (1993).

5. McLean, M. et al. A placental clock controlling the length of human pregnancy. Nature Med. 1, 460-463 (1995).

6. Thorburn, G.D. \& Rice, G.E. Placental PGE2 and the initiation of parturition in the sheep. in Eicosanoids in Reproduction (ed. Mitchell, M.D.) 73-86 (CRC Press, Boca Raton, Florida, 1990).

7. Wolfe, C.D.A. et al. Plasma corticotrophinreleasing factor (CRF) in abnormal pregnancy. Brit. J. Obstet. Gyn. 95, 1003-1006 (1988).

8. Challis, J.R.G. \& Hooper, S. Birth: Outcome of a positive cascade. in Perinatal Endocrinology (ed. Jones, C.T.) 781-793 ( Saunders, New York, 1989).

9. Warren, W.B., Patrick, S.L. \& Goland, R.S. Elevated maternal plasma corticotropin- releasing hormone levels in pregnancies complicated by pre-term labour. Am. J. Obstet. Gynecol. 166, 1198-1204 (1992).

Lawson Research Institute

St. Joseph's Health Centre

London, Ontario N6A 4V2, Canada 